\title{
Time Scales of the Trade Wind Boundary Layer Adjustment
}

\author{
GILLES BELLON \\ Centre National de Recherches Météorologiques, Météo-France, Toulouse, France \\ BJORN STEVENS \\ Max-Planck-Institut für Meteorologie, Hamburg, Germany
}

(Manuscript received 2 August 2012, in final form 15 November 2012)

\begin{abstract}
The adjustment of the trade wind atmospheric boundary layer to an abrupt sea surface warming is investigated using a large-eddy simulation (LES) and two simple bulk models: a mixed-layer model (MLM), and a model based on the mixing-line hypothesis (XLM). The near-surface temperature adjusts in a few hours, faster than can be expected from the characteristic time scales associated with the physical processes at play. The near-surface humidity adjusts more slowly, with a time scale of about a day, and it exhibits an initial decrease before increasing to its equilibrium value. An analysis of the MLM suggests that the initial tendency of humidity and temperature results from the difference in Bowen ratios between the equilibrium and the perturbation. An analysis of the three linear modes of the XLM shows that the fastest-decaying mode adjusts the subcloud-layer buoyancy, with a constructive interaction of all of the physical processes. The secondfastest-decaying mode is an adjustment of the boundary layer thermodynamical structure and the slowest mode adjusts the boundary layer depth. Approximate analytical expressions of the time scales characterizing these linear modes are derived both for the MLM and the XLM. The MLM exhibits no scale separation between the fastest and second-fastest time scales and a scale separation between these and the slowest time scale only in the case of a shallow well-mixed boundary layer. The XLM exhibits a scale separation between the buoyancy adjustment of the subcloud layer and the overall thermodynamic adjustment, while conserving the scale separation with the slower adjustment of the boundary layer depth.
\end{abstract}

\section{Introduction}

The trade wind atmospheric boundary layer can be considered in a Lagrangian perspective as vertical columns that move equatorward over progressively warmer water. This perspective has been used to help design field experiments [e.g., Atlantic Stratocumulus Transition Experiment (ASTEX) Lagrangian experiments; Albrecht et al. 1995] and in model intercomparisons (Bretherton et al. 1999; Sandu and Stevens 2011). From this Lagrangian point of view, the trade wind boundary layer is in permanent adjustment toward the equilibrium at the instantaneous sea surface temperature (SST). It is therefore of interest to investigate the characteristics of this adjustment and, in particular, the time scales involved.

Corresponding author address: Gilles Bellon, Centre National de Recherches Météorologiques, 42, avenue Gaspard Coriolis, 31057 Toulouse, France.

E-mail: gilles.bellon@meteo.fr
This can be done using idealized model experiments in which the SST is abruptly increased.

In the case of the stratocumulus-topped boundary layer (SCuBL), the adjustment time scales have been clearly documented in simple models as well as in a large-eddy simulation (LES). In both cases, the wellmixed thermodynamic variables adjust much faster than the depth of the layer, with a clear scale separation. In a simple mixed-layer model, Schubert et al. (1979b) found that the thermodynamic variables adjust with a time scale $\tau_{\text {th }}=\left(C_{d} V_{s} / h+D\right)^{-1} \approx 0.5$ day (with $h$ the characteristic depth of the boundary layer, $C_{d}$ the drag coefficient, $V_{s}$ the surface wind, and $D$ the divergence), and the inversion height adjusts on a time scale $\tau_{i}=D^{-1} \approx$ 5 days. Bretherton et al. (2010) found similar time scales in a mixed-layer model with a different closure and also in an LES; they showed that the scale separation is associated with the existence of slow manifolds.

Adjustment time scales have been investigated in deep convection (Tompkins and Craig 1998), albeit somewhat 
more heuristically, but there is very little in the literature about the time scales of the shallow-convective, cumulustopped boundary layer (CuBL). Betts (1993) evaluated that the adjustment of the subcloud layer alone to surface fluxes has a characteristic time scale of 0.6 day. Bretherton and Park (2008) described an LES experiment with perturbed SST and their analysis suggests a scale separation between the adjustment of thermodynamic variables and the adjustment of the CuBL depth similar to the case of the SCuBL. The LES behavior was also well reproduced by their bulk model.

For the CuBL, three basic time scales can be identified, each associated with a single physical process: the surface-flux time scale $\tau_{s}=h /\left(C_{d} V_{s}\right)$, the dynamical time scale $\tau_{D}=1 / D$, and the radiative time scale $\tau_{R}=$ $\Delta \theta / R$ [with $R$ the radiative cooling and $\Delta \theta$ a typical temperature contrast, such as the lower-tropospheric stability (LTS)]. For a typical CuBL, the typical time scales are $\left(\tau_{s}, \tau_{D}, \tau_{R}\right)=(1,2,4)$ days. The characteristic time scales of the CuBL are expected to be these time scales or combinations of these, such as $\tau_{\text {th }}$ and $\tau_{i}$ identified for the SCuBL (Schubert et al. 1979b). We thus expect characteristic time scales with orders of magnitudes of tens of hours to days. However, using LES to explore the response of the CuBL to a sudden change in SST shows that very short time scales are evident and that the initial adjustment proceeds in a nonintuitive manner.

In this study, we try to understand this adjustment of the CuBL. To do so, we perform an idealized LES with an abrupt SST change, and we interpret the results in the light of simple bulk models. Section 2 presents the framework used to define the large-scale conditions and the LES and bulk models used in this study. Section 3 presents the different model simulations and demonstrates that the simple bulk models reproduce important and nontrivial aspects of the LES response. Section 4 presents a more in-depth analysis of the time scales defined by the basic structure of our bulk models, followed by conclusions in section 5 .

\section{Framework and models}

\section{a. Idealized large-scale conditions}

We use the LES and bulk models presented in the next subsections integrated in the idealized framework developed by Bellon and Stevens (2012). This framework formulates the large-scale conditions and forcings in terms of a minimum set of parameters. The subsidence profile follows an exponential of altitude:

$$
w(z)=-w_{0}\left(1-e^{-z / z_{w}}\right),
$$

TABLE 1. Large-scale conditions and forcings.

\begin{tabular}{cccccc}
\hline \hline$w_{0}$ & $z_{w}$ & $R$ & $q_{0}$ & $\theta_{0}$ & $U$ \\
\hline $7.5 \mathrm{~mm} \mathrm{~s}^{-1}$ & $1200 \mathrm{~m}$ & $2 \mathrm{~K} \mathrm{day}^{-1}$ & $4 \mathrm{~g} \mathrm{~kg}^{-1}$ & $302.8 \mathrm{~K}$ & $10 \mathrm{~m} \mathrm{~s}^{-1}$ \\
\hline
\end{tabular}

where $w(z)$ is the large-scale vertical velocity at altitude $z, w_{0}$ is the high-altitude asymptotic value of subsidence ( $w_{0}>0$ for subsidence), and $z_{w}$ is a typical vertical scale of large-scale dynamics. The divergence at the surface is $D_{0}=w_{0} / z_{w}$ and it decreases exponentially with altitude.

The large-scale horizontal advection of humidity is neglected and radiation and large-scale horizontal advection of energy are modeled by a prescribed, altitudeindependent cooling $R$ ( $R>0$ for cooling). The vertical gradients of free-tropospheric humidity $q_{\mathrm{ft}}$ and potential temperature $\theta_{\mathrm{ft}}$ are constrained by the water balance $w \partial_{z} q_{\mathrm{ft}}=0$ and energy balance $w \partial_{z} \theta_{\mathrm{ft}}=-R$ in the nonturbulent troposphere, so that the free-tropospheric humidity and potential temperature profiles are determined by a reference humidity and temperature:

$$
\begin{aligned}
& q_{\mathrm{ft}}(z)=q_{0} \text { and } \\
& \theta_{\mathrm{ft}}(z)=\frac{R}{w_{0}} z_{w} \ln \left(e^{z / z_{w}}-1\right)+\theta_{0},
\end{aligned}
$$

with $q_{0}$ and $\theta_{0}$ two parameters that prescribe the uniform free-tropospheric specific humidity and the reference temperature at $z=z_{w} \ln (2)$. Equation (3) describes a profile that asymptotically approaches a linear profile at high altitude $\left(\partial_{z} \theta_{\mathrm{ft}} \rightarrow R / w_{0}\right)$, and tends toward infinitely cold temperatures at the surface.

The SST is set to a constant, $T_{s}$, and a constant geostrophic zonal wind $U$ is imposed (the latitude is set to $20^{\circ} \mathrm{N}$ ). The parameters describing the large-scale conditions and forcings are summarized in Table 1.

Using this framework, Bellon and Stevens (2012) studied the sensitivity of the equilibrium boundary layer to SST and free-tropospheric conditions, and Nuijens and Stevens (2012) studied the CuBL sensitivity to perturbations in the geostrophic wind speed. Here, we focus on the transient behaviors of the model. We present results for simulations in which $T_{s}$ is instantaneously increased from 298 to $299 \mathrm{~K}$ at $t=0$. The final state of the stationary simulation with $T_{s}=298 \mathrm{~K}$ presented in Bellon and Stevens (2012) is used as initial conditions and $T_{s}$ is set to $299 \mathrm{~K}$. We also performed simulations with lower and higher values of $T_{s}$ and found very similar behaviors. A few LES were performed in which the $T_{s}$ was decreased, and it was found that if the abrupt change is large enough (e.g., from 300 to $299 \mathrm{~K}$ ), the model behavior is highly nonlinear, going through a cloud-free regime before the shallow convection is reestablished. If 
the abrupt change is smaller (e.g., from 299.5 to $299 \mathrm{~K}$ ), the model behavior is very similar to the results presented here.

\section{b. LES}

We use the University of California, Los Angeles (UCLA) LES in a nonprecipitating configuration. The basic code is described by Stevens et al. (2005), who solve the Ogura-Phillips anelastic equations using finite differences on a regular-horizontal, stretchedvertical mesh. Cloud and microphysical processes are represented following the procedures described by Savic-Jovcic and Stevens (2008).

Simulations are performed over a square horizontal domain of $6.4 \mathrm{~km} \times 6.4 \mathrm{~km}$, with a horizontal resolution of $50 \mathrm{~m}$ in both directions. The vertical grid consists of 120 vertical levels with a 10 -m grid spacing at the surface increasing by $2 \%$ at each level so that the domain top is at $4830 \mathrm{~m}$.

With the large-scale conditions used in the present work, the boundary layer is not very deep and the nonprecipitating assumption seems reasonable. But precipitation could have an influence on the transient behavior of the models, in particular via the precipitation flux at cloud base. We do not address this issue in the present work.

\section{c. Mixing-line model}

The mixing-line model (XLM) is fully described in Bellon and Stevens (2012). It is a bulk model of the trade wind boundary layer based on the work of Betts and Ridgway (1988). Profiles of conserved variables above the cloud base are described as linear mixtures between the subcloud and inversion-top air. The mixing line is defined as the height-dependent ratio of inversion-top air in an air parcel at a given altitude. (The rest of the parcel is considered to originate from the subcloud layer.) In the bulk formulation of the mixing-line model, only the cloud-layer vertical average of the mixing line, which we denote by $\alpha$, and its divergence-weighted vertical average, which we denote by $\gamma$, appear in the bulk water and energy budgets. In Bellon and Stevens (2012), these parameters are tuned using the stationary states of LES with varying surface and free-tropospheric conditions; here we use the same values of these parameters: $\alpha=0.35$ and $\gamma=0.8$. The XLM was also generalized to model cloud-free boundary layers as well as cloudy ones, but here we focus on the CuBL. We reformulate the XLM in this regime [Eqs. (15a) and (16)(18) in Bellon and Stevens (2012)] to express the equations in terms of total water $q_{t}$ and $\theta_{v}=\theta_{l}+\epsilon_{1} \theta_{r} q_{t}$, where $\theta_{l}$ is the liquid water potential temperature, $\theta_{r}$ is a reference temperature $(299 \mathrm{~K})$, and $\epsilon_{1}=R_{v} / R_{d}-1$, with $R_{d}$ (respectively, $R_{v}$ ) the gas constant of dry air (respectively, water vapor). In the absence of condensate, $\theta_{v}$ is the virtual potential temperature; in the cloud layer, it is a crude measure of buoyancy and we will refer to it as pseudobuoyancy.

In this formulation, the prognostic variables of the XLM are the altitude of the inversion-top $h$, also referred to as the CuBL depth, and two conserved variables in the subcloud well-mixed layer: the virtual potential temperature $\theta_{v M}$ and total water mixing ratio $q_{M}$. The altitude of cloud-base $\eta$ is a fourth, diagnostic variable. The equations of the XLM in the shallowconvective regime are the water and energy budgets of the $\mathrm{CuBL}$ and two closures at cloud base. For the largescale conditions described above, the vertically integrated water and pseudobuoyancy budgets are

$$
\begin{aligned}
\partial_{t}\left(h\left\langle q_{t}\right\rangle\right)-\partial_{t} h q_{0}= & \gamma w_{h}\left(q_{0}-q_{M}\right) \\
& +w_{s}\left(q_{s}-q_{M}\right) \text { and } \\
\partial_{t}\left(h\left\langle\theta_{v}\right\rangle\right)-\partial_{t} h \theta_{v f \mathrm{ft}}(h)= & \gamma w_{h}\left[\theta_{v \mathrm{ft}}(h)-\theta_{v M}\right] \\
& +w_{s}\left(\theta_{v s}-\theta_{v M}\right)-R h,
\end{aligned}
$$

with the inversion-top subsidence $w_{h}=-w(h)$ and the characteristic speed of surface fluxes $w_{s}=C_{d} U$, with $C_{d}=1.2 \times 10^{-3}$. The saturation mixing ratio at temperature $T$ and pressure $p$ is noted $q^{*}(T, p) ; q_{s}=q^{*}\left(T_{s}, p_{s}\right)$ is the saturated mixing water ratio at the surface, with $p_{s}$ the surface pressure, and $\theta_{v s}$ is the surface virtual potential temperature: $\theta_{v s}=T_{s}\left(p_{0} / p_{s}\right)^{R_{d} / c_{p}}+\epsilon_{1} \theta_{r} q_{s}$ with $p_{0}$ the reference pressure and $c_{p}$ the specific isobaric heat capacity of dry air. Angle brackets indicate the vertical average over the CuBL:

$$
\langle\phi\rangle=\phi_{M}+\alpha\left(1-\frac{\eta}{h}\right)\left[\phi_{i^{+}}(h)-\phi_{M}\right]
$$

where $\phi$ is either $q_{t}$ or $\theta_{v}$.

In Eqs. (4) and (5), the left-hand side corresponds to the rate of change of the vertically integrated water or energy content of the CuBL. On the right-hand side, the first terms correspond to the vertical advections by the subsidence $w_{h}$, with the parameter $\gamma$ describing the effect of the mixing line compared to a well-mixed boundary layer (which corresponds to $\gamma=1$ ). The second terms correspond to the surface fluxes, and the third term in Eq. (5) is the prescribed horizontal advection and radiation.

The first closure at cloud base assumes that the turbulent buoyancy flux $F^{v}$ at cloud base is proportional to the surface buoyancy flux: $F^{v}(\eta)=-k F^{v}(0)$, with a factor $k=0.2$. This closure is not expected to be valid instantaneously after an abrupt change in $T_{s}$ and associated 
surface fluxes but rather to adjust on a time scale characteristic of the eddy turnover. From our LES as well as the one documented in Bretherton and Park (2008), it appears that the turbulence and cloud mass flux adjusts within a few minutes of the SST change. This time scale is small compared to the time scales of at least a few hours under consideration here, and we apply the closure instantaneously. It can be used to simplify the buoyancy budget of the subcloud layer:

$$
\eta \partial_{t} \theta_{v M}=(1+k) w_{s}\left(\theta_{v s}-\theta_{v M}\right)-R \eta
$$

The second closure assumes that the cloud base is at the lifting condensation level (LCL):

$$
q_{M}=q^{*}[T(\eta), p(\eta)]
$$

\section{d. Mixed-layer model}

The mixed-layer model (MLM) is used in Bellon and Stevens (2012) as well. It models the boundary layer as a well-mixed layer capped by an infinitely thin inversion, following the work of Lilly (1968) and Schubert et al. (1979a,b). The equations of the MLM are based on the water and energy budgets of this layer, and one closure on the downward pseudobuoyancy flux at the base of the inversion, which is considered as proportional to the surface upward buoyancy flux (with a factor $k=0.2$ ). This simplification is the main difference with previous mixed-layer models starting with that in Schubert et al. (1979a,b). With our large-scale conditions, the resulting equations are as follows:

$$
\begin{aligned}
\partial_{t}\left(h q_{M}\right)-\partial_{t} h q_{0}= & w_{h}\left(q_{0}-q_{M}\right)+w_{s}\left(q_{s}-q_{M}\right), \\
\partial_{t}\left(h \theta_{v M}\right)-\partial_{t} h \theta_{v f \mathrm{t}}(h)= & w_{h}\left[\theta_{v \mathrm{ft}}(h)-\theta_{v M}\right] \\
& +w_{s}\left(\theta_{v s}-\theta_{v M}\right)-R h, \quad \text { and }
\end{aligned}
$$

$$
h \partial_{t} \theta_{v M}=(1+k) w_{s}\left(\theta_{v s}-\theta_{v M}\right)-R h .
$$

These equations are identical to the XLM equations with $\alpha=0, \gamma=1$, and $\eta=h$ instead of the closure on the LCL. This type of model was developed to simulate the SCuBL or the convective boundary layer. Although it is not capable of representing the dependence of the stationary states of the $\mathrm{CuBL}$ as a function of changes in the large-scale conditions, we use it here to explore the initial evolution of the subcloud layer, as it provides some insight into the behavior of the more complex models and the LES.

\section{Results}

\section{a. LES results}

Figure 1 shows the time evolution of $h, \eta, q_{M}$, and $\theta_{M}$, latent and sensible turbulent heat fluxes at the surface and at cloud base, and the cloud fraction for the experiment described in the previous section ( $T_{s}$ is increased from 298 to $299 \mathrm{~K}$ at $t=0$ ).

The adjustment of $\theta_{M}$ appears to be much faster than the other variables, with an adjustment time of a couple of hours (Fig. 1c). The sensible heat flux (SHF) at the surface and at cloud base and cloud-base latent heat flux (LHF) adjust fast as well (Figs. 1d,e). This adjustment happens on time scales that are significantly shorter than the time scales $\tau_{s}, \tau_{D}$, and $\tau_{R}$ associated with the physical processes, or a simple combination of them such the fast time scale $\tau_{\text {th }}$ identified in earlier studies (Schubert et al. 1979b; Bretherton et al. 2010), or even the surface-flux time scale of the subcloud layer (Betts 1993).

The subcloud mixing ratio and the LHF at the surface adjust somewhat slower (Figs. 1b,d) but still faster than $h$ (which is not yet at its stationary value of $1370 \mathrm{~m}$ after 8 days; see Fig. 1a); the time evolutions of $q_{M}$ and of the surface LHF are also nonmonotonic.

The initial decrease in $q_{M}$ results from the initial increase in cloud-base LHF that is stronger than the initial increase in the surface LHF (Fig. 1d); the surface LHF increases at $t=0$ because of the abrupt change in $T_{s}$, and it then increases further because of the decrease in $q_{M}$. The increase in the cloud-base LHF results from an increase in turbulence associated with the increase in surface buoyancy flux, which drives more cloud mixing as suggested by the increase in cloud fraction (Fig. 1f). The increased turbulence at cloud base warms and dries the subcloud layer.

\section{b. XLM results}

The XLM exhibits the same behavior as the LES. This is shown in Fig. 2, which shows the same simulation as was performed by the LES, but with the XLM (thick lines in Figs. 2a-c, all lines in Figs. 2d,e).

As was also evident in the LES, the LHF increases more at cloud base than at the surface for complex reasons. One key element is that the strong increase in surface buoyancy flux is driven by the increase in surface SHF (a buoyancy flux increase of $12 \mathrm{~W} \mathrm{~m}^{-2}$ ) rather than by the surface LHF (a buoyancy flux increase of $3 \mathrm{~W} \mathrm{~m}^{-2}$ ). This buoyancy flux results in strong mixing at cloud base.

\section{c. MLM interpretation of the initial tendencies of temperature and humidity}

We can try to understand the initial warming and drying of the CuBL with the MLM. Our approach is to 

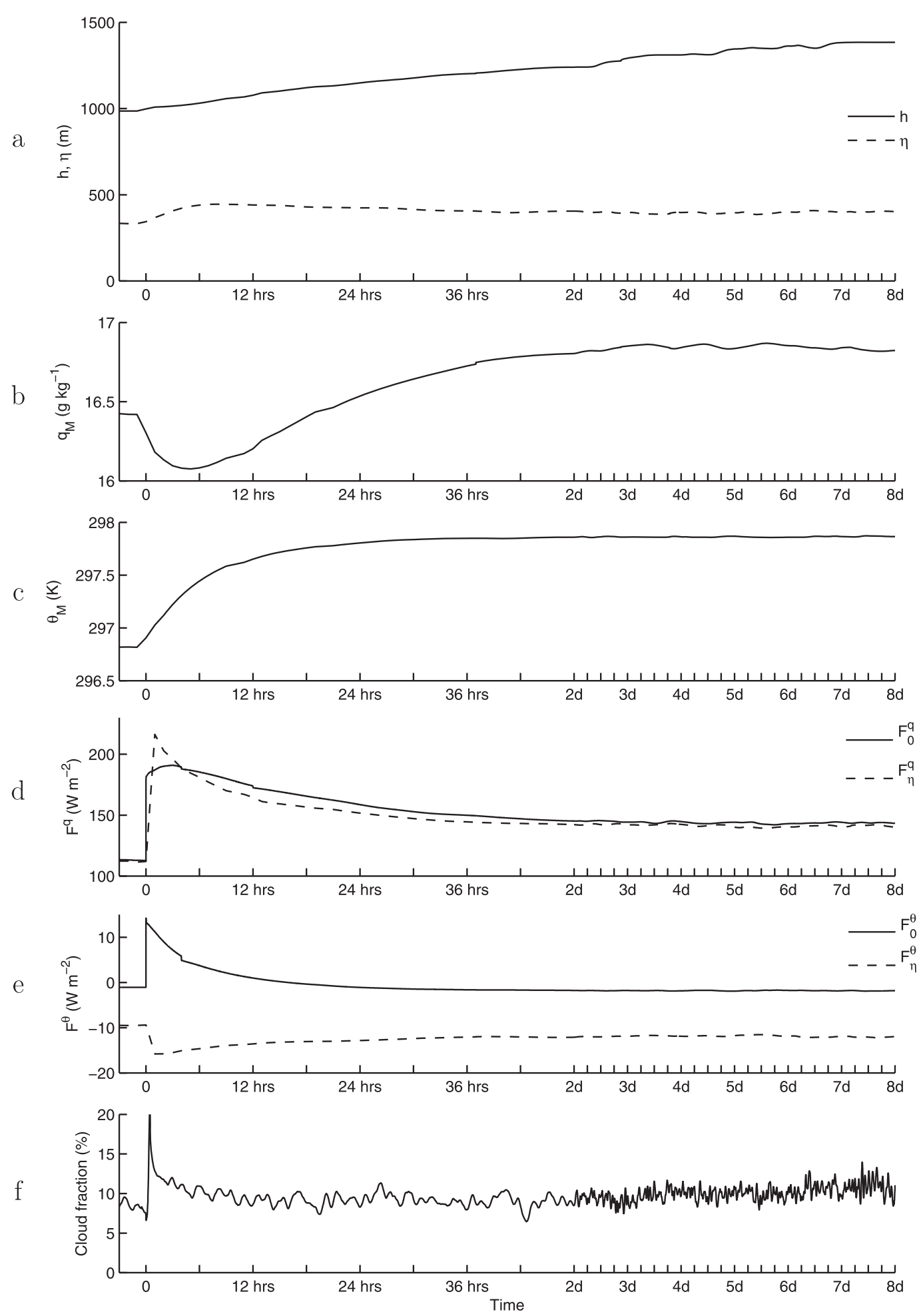

FIG. 1. Time evolution of (a) the altitudes of the inversion top (solid line) and of the cloud base (dashed), (b) subcloud water ratio, (c) subcloud potential temperature, (d) latent and (e) sensible heat fluxes at the surface and cloud base, and (f) cloud fraction for the first 8 days of the LES with $T_{s}=299 \mathrm{~K}$, using the stationary state with $T_{s}=$ $298 \mathrm{~K}$ as initial conditions. Note that the horizontal time scale changes after 2 days. 
a

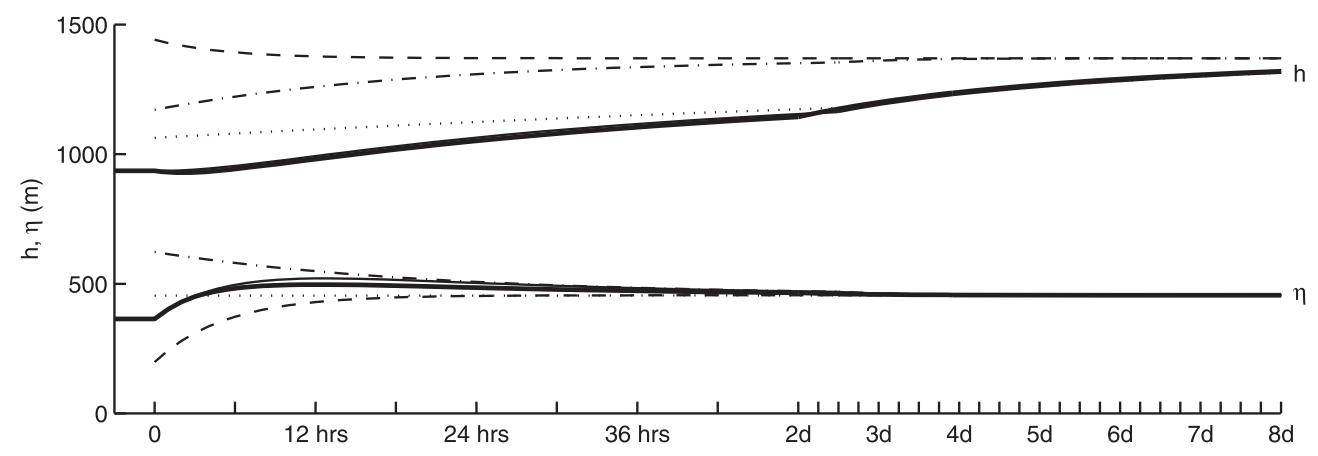

$\mathrm{b}$
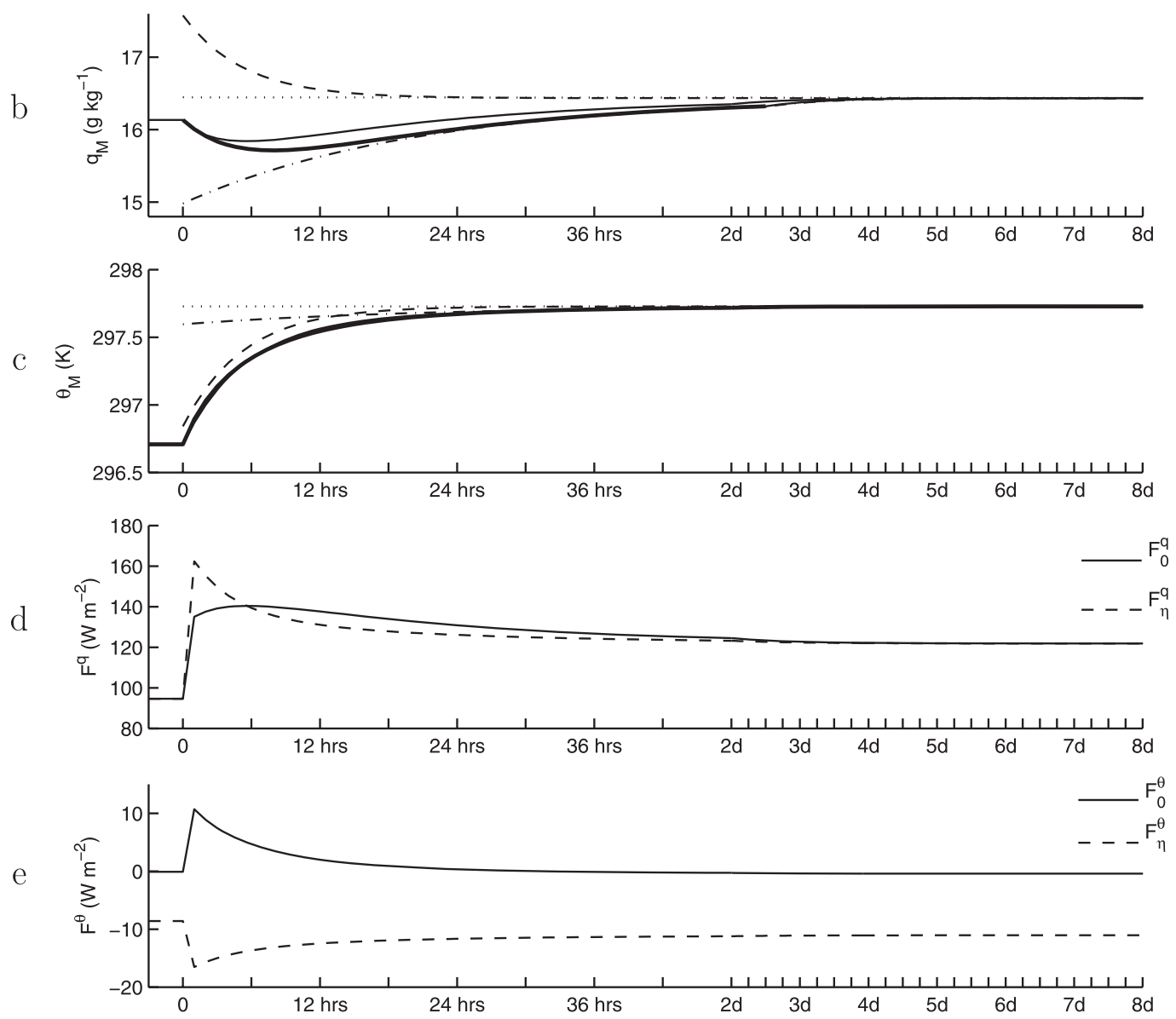

FIG. 2. Time evolution of (a) the altitudes of the inversion top and of the cloud base, (b) subcloud water ratio, (c) subcloud potential temperature, and (d) latent and (e) sensible heat fluxes at the surface (solid lines) and cloud base (dashed) for the first 8 days of the XLM experiment with $T_{s}=299 \mathrm{~K}$, using the stationary state with $T_{s}=$ $298 \mathrm{~K}$ as initial conditions. In (a)-(c), the nonlinear experiment is shown by the thick solid lines, the linear time evolution is shown by the thin solid lines, and the latter's projections on mode 1 , mode 2 , and mode 3 are shown, respectively, by the dashed, dashed-dotted, and dotted lines. Note that the horizontal time scale changes after 2 days.

explore the initial response of the MLM about the initial state, which is the equilibrium state before the abrupt change in $T_{s}$. To do so we first solve for the equilibrium state before the abrupt change in $T_{s}$, and then use this to derive an equation for the initial tendencies of the boundary layer temperature and moisture, to see if the counterintuitive drying can be represented by this model.

If, at $t=0$, the model is at equilibrium with $T_{s}=$ $298 \mathrm{~K}$, we can write Eq. (9) and the difference between 
Eqs. (10) and (11) (which is actually the closure at the inversion that simplifies the buoyancy budget of the infinitely thin inversion layer):

$$
\begin{aligned}
& 0=w_{\bar{h}}\left(q_{0}-\bar{q}_{M}\right)+w_{s}\left(q_{s}-\bar{q}_{M}\right) \quad \text { and } \\
& 0=w_{\bar{h}}\left[\theta_{v \mathrm{ft}}(\bar{h})-\bar{\theta}_{v M}\right]-k w_{s}\left(\theta_{v s}-\bar{\theta}_{v M}\right),
\end{aligned}
$$

with the overbar indicating the equilibrium with $T_{s}=298 \mathrm{~K}$.

If at $t=0, T_{s}$ is increased by an increment $T_{s}^{\prime}$, the initial tendency of the boundary layer virtual potential temperature can be obtained from Eq. (11):

$$
\bar{h} \partial_{t} \theta_{v M}(t=0)=(1+k) w_{s} \theta_{v s}^{\prime},
$$

where $\theta_{v s}^{\prime}=T_{s}^{\prime}\left(p_{0} / p_{s}\right)^{R_{d} / c_{p}}+\epsilon_{1} \theta_{r} q_{s}^{\prime}$ is the perturbation of surface virtual potential temperature resulting from $T_{s}^{\prime}$, with $q_{s}^{\prime}$ the surface saturation mixing ratio perturbation resulting from $T_{s}^{\prime}$ (which is of the same sign as $T_{s}^{\prime}$ ). For a warming, the initial tendency of $\theta_{v M}$ is positive.

Additionally, Eq. (9) can be rewritten introducing the CuBL-top entrainment speed $w_{e}=\partial_{t} h+w_{h}$ to express the initial tendency in water mixing ratio:

$$
\bar{h} \partial_{t} q_{M}(t=0)=w_{e}\left(q_{0}-\bar{q}_{M}\right)+w_{s} q_{s}^{\prime} .
$$

The closure at the inversion provides an expression of $w_{e}$ :

$$
0=w_{e}\left[\theta_{v \mathrm{ft}}(\bar{h})-\bar{\theta}_{v M}\right]-k w_{s} \theta_{v s}^{\prime},
$$

which can be introduced in Eq. (15), while using Eqs. (12) and (13) to replace the ratio of the equilibrium jumps at the inversion by the ratio of the equilibrium sea-air contrasts at the surface:

$$
\bar{h}_{t} q_{M}(t=0)=w_{s}\left(q_{s}^{\prime}-\theta_{v s}^{\prime} \frac{q_{s}-\bar{q}_{M}}{\theta_{v s}-\bar{\theta}_{v M}}\right) .
$$

The sign of the initial tendency in humidity depends on the competition between the perturbations of evaporation and the perturbation of surface buoyancy flux weighted by the ratio of air-sea humidity contrast to airsea virtual potential temperature contrast. This can be written as a simple dependence on the Bowen ratio $B^{\prime}$ of the initial perturbation (defined as the ratio of the sensible flux anomaly to the latent flux anomaly) and on the equilibrium Bowen ratio $\bar{B}$ :

$$
\bar{h} \partial_{t} q_{M}(t=0)=w_{s} q_{s}^{\prime}\left(1-\frac{c+B^{\prime}}{c+\bar{B}}\right)
$$

with the constant $c=\epsilon_{1} \theta_{r} c_{p} / L_{v}\left(p_{s} / p_{0}\right)^{R_{d} / c_{p}} \approx 0.08, L_{v}$ being the latent heat of vaporization.
Through the closure on the buoyancy flux, the equilibrium surface sensible heat flux is constrained by the ratio of the mixed-layer radiative cooling over the surface latent heat flux: $(1+k)(c+\bar{B})=\Delta F^{R} / \mathrm{LHF}$, with $\Delta F^{R}$ the divergence of the radiative flux across the layer. Since the radiative cooling is about $30 \mathrm{~W} \mathrm{~m}^{-2}$ compared to a large evaporation of about $150 \mathrm{~W} \mathrm{~m}^{-2}, \bar{B}$ is about 0.1 , which is a typical value for the tropical boundary layer. The Bowen ratio of the initial perturbation associated with a change of $T_{s}$ at constant pressure is typically 0.4 , so that the term in parentheses in Eq. (18) is negative. The initial tendency of $q_{M}$ therefore has the opposite sign to the initial perturbation of evaporation $w_{s} q_{s}^{\prime}$ and to the initial tendency of $\theta_{M}$. Ultimately, these opposite signs result from the fact that the initial surface SHF perturbation associated with a change in surface temperature is comparable to the corresponding LHF perturbation, but the equilibrium SHF is small compared to the equilibrium LHF because of the constraints of the energy and water balances of the mixed layer. As a result, an increase in SST increases evaporation less than it increases entrainment drying through the increase in inversion-base buoyancy flux. This result is dependent on the closure used in the MLM: Schubert et al. (1979b), using a more refined closure, did not obtain such opposite tendencies.

Although the vertical structure and closures are different in the XLM and LES, the initial drying of the subcloud layer in our experiment suggests that a similar mechanism is at play. We can consider that the relevant processes in the cloud and inversion layers of the XLM and LES behave similarly to the processes controlling the infinitely thin inversion layer in the MLM. In the XLM and LES, the SST increase causes an increase in buoyancy flux at cloud base (in absolute value); the resulting increase in cloud turbulence increases entrainment at the inversion, and entrainment drying is efficiently communicated to the subcloud layer by the enhanced cloud turbulence. For the XLM, we have not found simple analytical expressions for the initial tendencies because of the increased complexity in the vertical structure, so in the next section we approach this through a more formal linearization.

\section{d. Linear XLM results}

We can linearize the system of Eqs. (4)-(8) around a reference equilibrium state and replace $\eta$ in Eqs. (4)(7) using Eq. (8), and nondimensionalize the system using the following scales:

$$
\begin{aligned}
& {[z]=(1-\alpha) \bar{h}+\alpha \bar{\eta},} \\
& {[q]=\bar{q}_{M}-q_{0},}
\end{aligned}
$$


TABLE 2. Nondimensional parameters for a reference equilibrium state with $T_{s}=299 \mathrm{~K}$.

\begin{tabular}{ccccccccccc}
\hline \hline$\tilde{\eta}$ & $\Gamma$ & $\Theta$ & $Q$ & $\epsilon_{w}$ & $\epsilon_{R}$ & $\delta$ & $\lambda$ & $\mu$ & $\nu$ & $\psi$ \\
\hline 0.45 & 0.74 & 0.71 & 1.84 & 0.4 & 0.32 & 0.39 & 0.30 & 0.52 & 0.52 & -0.02 \\
\hline
\end{tabular}

$$
\begin{aligned}
& {[\theta]=\theta_{v i^{+}}(\bar{h})-\bar{\theta}_{v M} \text {, and }} \\
& {[t]=\frac{[z]}{w_{s}} .}
\end{aligned}
$$

These scalings are dependent on the reference state indicated by the overbar. For a reference state that corresponds to the model equilibrium for $T_{s}=299 \mathrm{~K},[z] \approx$ $1 \mathrm{~km}$ is the equivalent height of the CuBL that characterizes the change of the CuBL vertically integrated heat or water content associated with a change in the subcloud variable, $[q] \approx 12 \mathrm{~g} \mathrm{~kg}^{-1}$ and $\left[\theta_{v}\right] \approx 8 \mathrm{~K}$ characterize the stratification accross the CuBL, and $[t] \approx 22 \mathrm{~h}$ is the time scale associated with surface fluxes.

The linearized, nondimensionalized system can be written in a matrix formulation:

$$
\mathbf{P} \cdot \partial_{\hat{t}} \mathbf{X}=\mathbf{M} \cdot \mathbf{X},
$$

with $\mathbf{X}$ the state vector

$$
\mathbf{X}=\left[\begin{array}{c}
\hat{h} \\
\hat{q}_{M} \\
\hat{\theta}_{v M}
\end{array}\right]
$$

where the circumflex indicates the nondimensional perturbation. Using Eqs. (4)-(8), we can write the matrices $\mathbf{P}$ and $\mathbf{M}$ as follows:

$$
\begin{aligned}
& \mathbf{P}=\left[\begin{array}{ccc}
1-\alpha & 1-\alpha Q & \alpha \Theta \\
-1+\alpha(1+\Gamma) & \alpha Q & 1-\alpha \Theta \\
0 & 0 & \tilde{\eta}
\end{array}\right] \text { and } \\
& \mathbf{M}=-\left[\begin{array}{ccc}
\delta \gamma \epsilon_{w} & 1+\gamma \epsilon_{w} & 0 \\
(1-\gamma) \epsilon_{R}-\delta \gamma \epsilon_{w} & 0 & 1+\gamma \epsilon_{w} \\
0 & -Q \epsilon_{R} & 1+k+\Theta \epsilon_{R}
\end{array}\right],
\end{aligned}
$$

with the following notations: $\tilde{\eta}=\bar{\eta} /[z]$ is the normalized reference depth of the subcloud layer, $\Gamma=\partial_{z} \theta_{i^{+}}(\bar{h})[z] /[\theta]$ is the normalized reference gradient of potential temperature at the top of the inversion, $\epsilon_{w}=w_{\bar{h}} / w_{s}$ is the nondimensional rate of subsidence there, $\epsilon_{R}=R / w_{s} \cdot[z] /[\theta]$ is the nondimensional rate of radiation, and $\delta=$ $\partial_{z} w(\bar{h})[z] / w_{\bar{h}}$ is the nondimensional ratio between divergence and subsidence at the inversion top. Here $\Theta$ $(Q)$ is the nondimensional rate of increase (decrease) of $\eta$ with $\theta_{v M}\left(q_{M}\right)$ :

$\Theta=\frac{\partial \eta}{\partial \theta_{v M}}\left(\bar{q}_{M}, \bar{\theta}_{v M}\right) \frac{[\theta]}{[z]}$ and $Q=-\frac{\partial \eta}{\partial q_{M}}\left(\bar{q}_{M}, \bar{\theta}_{v M}\right) \frac{[q]}{[z]}$

$\partial_{\theta_{v M}} \eta$ and $\partial_{q_{M}} \eta$ can be computed by differentiating Eq. (8) above [see, for example, appendix 3 in Bretherton and Park (2008)].

Table 2 lists the nondimensional parameters for the $T_{s}=299 \mathrm{~K}$ equilibrium as reference state.

In $\mathbf{M}$ [see Eq. (26)], the diagonal terms of the upperright block correspond to the effect of the surface turbulent fluxes and advection of anomalous humidity and temperature. The first coefficient on the first line corresponds to the additional advection due to the vertical displacement of the inversion top. The first coefficient on the second line corresponds to a combination of this additional subsidence with the additional radiative cooling and the change in the potential temperature of the advected air.

The matrix $\mathbf{P}$ admits the inverse

$$
\mathbf{P}^{-1}=\frac{1}{\tilde{\eta} \nu}\left[\begin{array}{ccc}
\alpha Q \tilde{\eta} & -(1-\alpha Q) \tilde{\eta} & \psi \\
\lambda \tilde{\eta} & (1-\alpha) \tilde{\eta} & -\mu \\
0 & 0 & \nu
\end{array}\right]
$$

where $\lambda=1-\alpha-\alpha \Gamma, \mu=1-\alpha-\alpha^{2} \Gamma \Theta, \nu=1-\alpha-$ $\alpha \Gamma(1-\alpha Q)$, and $\psi=1-\alpha(Q+\Theta)$.

The system (23) can be rewritten

$$
\partial_{\hat{t}} \mathbf{X}=\mathbf{N} \cdot \mathbf{X}, \text { with } \quad \mathbf{N}=\mathbf{P}^{-1} \cdot \mathbf{M} .
$$

Figures $2 \mathrm{a}-\mathrm{c}$ show the time evolution of the linear XLM with $T_{s}=299 \mathrm{~K}$ and an initial state that is the equilibrium solution for $T_{s}=298 \mathrm{~K}$ (solid thin lines). It shows that, although the nonlinearities are not negligible, the response of the nonlinear XLM is dominated by its linear component.

The linear response can be further projected onto the three eigenmodes of $\mathbf{N}$. This decomposition is shown in Fig. 2 as well (dashed, dashed-dotted, and dotted lines). The three modes have distinct characteristics: 


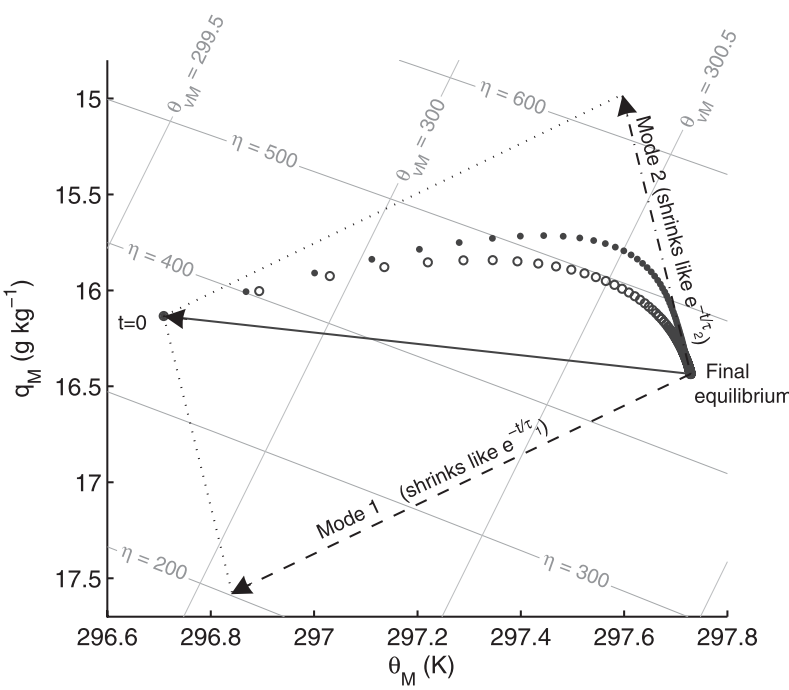

FIG. 3. Time evolution of the XLM model in the $\left(\theta_{M}, q_{M}\right)$ plan. Hourly results from the nonlinear and linear models are shown, respectively, by open and filled circles. The initial perturbation from the final equilibrium is shown as a solid arrow, and its decomposition along linear modes 1 and 2 are shown, respectively, by dashed and dashed-dotted arrows. The contribution of mode 3 is not shown because it is very small. Isolines of $\eta$ and $\theta_{v M}$ are also drawn.

- The fastest-decaying mode (mode 1 , dashed lines) is associated with a decay time scale of $\tau_{1} \approx 5 \mathrm{~h}$. It accounts for the fast adjustment of $\theta_{M}$, while it also accounts for the early decrease in $q_{M}$, because the potential temperature and total water perturbations associated with the corresponding eigenvector are of opposite sign. It is also associated with a deepening of the subcloud layer (owing to the change in LCL associated with the temperature and humidity perturbations) and a slight thinning of the CuBL.
- The second-fastest-decaying mode (mode 2, dasheddotted lines) is associated with a decay time scale of $\tau_{2} \approx 20 \mathrm{~h}$. It accounts for a residual warming of the subcloud layer that adjusts $\theta_{M}$ to its final equilibrium value as well as its moistening, which compensates the early drying and adjusts $q_{M}$ to its final equilibrium value. It is also associated with a thinning of the subcloud layer (owing to its moistening) and a deepening of the CuBL. - The slowest-decaying mode (mode 3 , dotted lines) is associated with a time scale of $\tau_{3} \approx 108 \mathrm{~h}$. It accounts for a large part of the growth of the CuBL. But it is not associated with any significant perturbation of the subcloud conserved variables. It accounts for the slow adjustment of the cloud and inversion layers.

The adjustment of the subcloud layer can also be described in the $\left(\theta_{M}, q_{M}\right)$ plan, as shown in Fig. 3. This figure shows that the nonlinearities of the XLM tend to limit the decrease in $q_{M}$ and hasten the adjustment at the beginning of the simulation. It also highlights that mode 1 adjusts most of the perturbation in $\theta_{M}$ and is responsible for the initial drying. Since mode 3 has a negligible role in the adjustment of the subcloud layer, the time evolution of $\theta_{M}$ and $q_{M}$ is essentially along mode 2 after $20 \mathrm{~h}$ of simulation, once the contribution of mode 1 to the perturbation has decayed. The isolines of $\eta$ show that the adjustment of the cloud-base altitude is the result of a compensation between mode 1 and mode 2 . The isolines of $\theta_{v M}$ show that its perturbation projects on mode 2 more than the $\theta_{M}$ perturbation does, so that the subcloud virtual temperature is adjusted slower than the temperature.

To better understand the time evolution of the subcloud layer, Fig. 4 shows the contributions of modes 1 and 2 to the perturbations of the terms in the subcloud water and energy budgets. The sum of these terms cause the time evolution of the perturbation $\theta_{M}$ and $q_{M}$
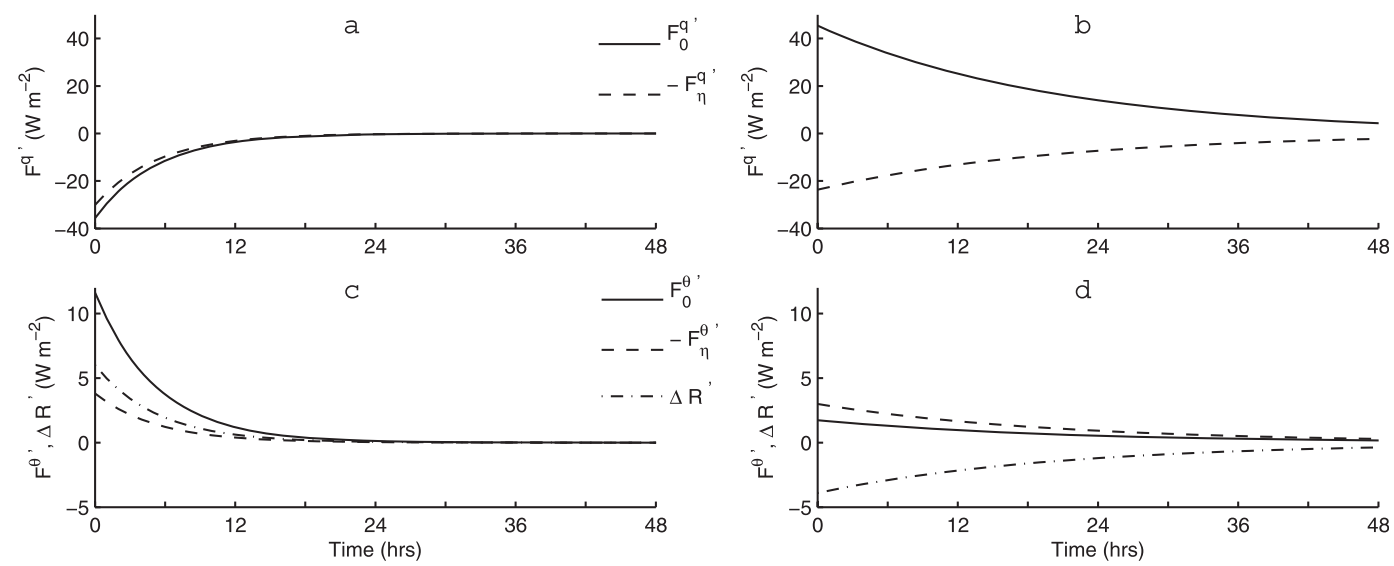

FIG. 4. Time evolution of the perturbations in the (a),(b) water and (c),(d) energy budgets associated with modes (a),(c) 1 and (b),(d) 2. Surface fluxes are shown by solid lines, the contribution of cloud-base fluxes are shown by dashed lines, and the net radiative contribution is shown by dashed-dotted lines. 


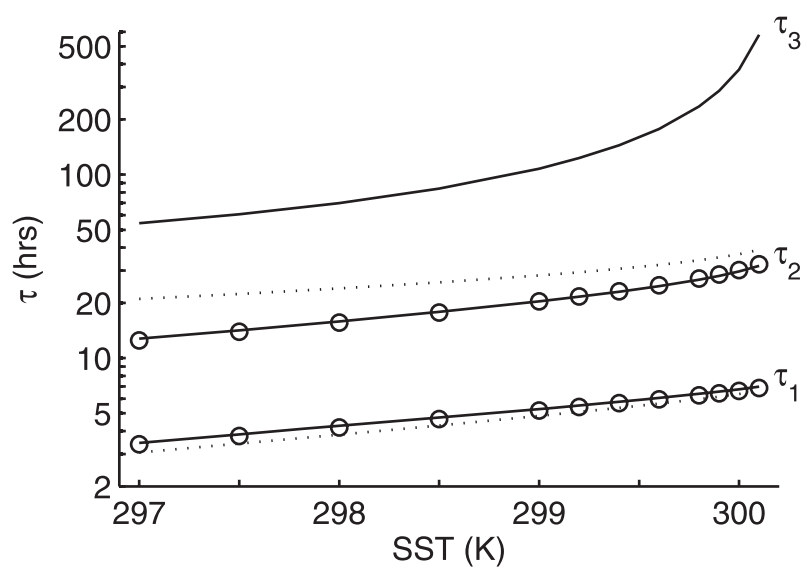

FIG. 5. Adjustment time scales as a function of the SST used to compute the reference equilibrium state (solid line). First-order time scales $\tau_{1}^{o}$ and $\tau_{2}^{o}$ (open circles) as well as their approximations with $\chi=0$ (dotted lines) are also shown.

associated to these modes in Figs. $2 \mathrm{a}$ and $2 \mathrm{~b}$. It is striking that all the terms in mode 1 tend to damp the humidity or temperature perturbation, while some compensation between terms occurs in mode 2 . This explains why mode 1 is damped faster than mode 2 .

- In mode 1 , the negative perturbation of $\theta_{M}$ causes an increase in surface SHF that warms the subcloud layer and powers more turbulence and a larger (negative) cloud-base SHF and a larger (positive) cloud-base LHF, which warm and dry the subcloud layer. These mechanisms are similar to those explaining the initial tendencies in the MLM (see previous section). The positive perturbation of $q_{M}$ also causes a negative anomalous surface LHF that dries the subcloud layer. The moist and cool subcloud anomalies also lower the LCL and shoal the subcloud layer, reducing the radiative cooling of the layer, so that the perturbation of the radiative contribution also warms the subcloud layer.

- In mode 2, the negative perturbations of both $\theta_{M}$ and $q_{M}$ cause an increase in surface SHF and LHF that damp the temperature and humidity perturbations. These fluxes in turn increase the cloud-base fluxes that warm and dry the subcloud layer, damping the temperature anomaly and opposing the reduction of the humidity anomaly. The contribution of the humidity perturbation to the change in LCL is larger than that of the temperature perturbation, so that the perturbation of the cloud-base altitude is positive and the perturbation of the radiative cooling is negative (more cooling), opposing the return to equilibrium.

This shows that the adjustment time scales depend on a complex interaction (constructive or destructive) between the processes rather than one or two processes only. This is the main reason why the time scales $\tau_{1}, \tau_{2}$, and $\tau_{3}$ do not have systematically the same order of magnitude as the process time scales $\tau_{D}, \tau_{s}$, and $\tau_{R}$.

\section{e. Sensitivity of the adjustment time scales to SST}

Figure 5 shows the sensitivity of the time scales $\tau_{n}$ to the equilibrium basic state. The sensitivity of the XLM equilibrium to SST was presented in Bellon and Stevens (2012). Here, we compute the corresponding adjustment time scales; these are quite sensitive to the reference state, with $\tau_{1}$ doubling from 3.5 to $7 \mathrm{~h}, \tau_{2}$ going from 13 to $32 \mathrm{~h}$, and $\tau_{3}$ growing tenfold from 54 to $578 \mathrm{~h}$ for a $3-\mathrm{K}$ increase in $T_{s}$. For $T_{s}$ slightly warmer than $300 \mathrm{~K}$, $\tau_{3}$ goes to infinity, which is consistent with the instability of the equilibrium described in Bellon and Stevens (2012) (the corresponding growth rate changes sign). Furthermore, it explains why the subcloud conserved variables are almost stationary in the unstable cases shown in Bellon and Stevens (2012): the subcloud layer adjusts rapidly thanks to the quick damping of modes 1 and 2, while the growth of the CuBL follows the pattern of mode 3 with little variation of the subcloud temperature and humidity.

\section{Analytical approximations to the adjustment time scales}

Since the adjustment time scales $\tau_{n}$ are very sensitive to the equilibrium, it is difficult to distinguish the contribution of each process to the time scales without taking into account the sensitivity of the equilibrium or imposing an unbalanced basic state. Fortunately, the adjustment time scales can be derived analytically, both for the MLM, and with some approximation for the XLM. Doing so provides insight into the physical processes behind the time scales and is the purpose of this section.

\section{a. Adjustment time scales of the MLM}

First, as a reference, we compute the characteristic time scales of the MLM. Similarly to the linear system of equations [Eq. (23)], the MLM system of Eqs. (9)-(11) can be linearized and nondimensionalized, and written in the following matrix form:

$$
\mathbf{P}_{m} \cdot \partial_{\hat{t}} \mathbf{X}=\mathbf{M}_{m} \cdot \mathbf{X},
$$

with

$$
\begin{aligned}
& \mathbf{P}_{m}=\left[\begin{array}{ccc}
1 & 1 & 0 \\
-1 & 0 & 1 \\
0 & 0 & 1
\end{array}\right] \text { and } \\
& \mathbf{M}_{m}=-\left[\begin{array}{ccc}
\delta \epsilon_{w} & 1+\epsilon_{w} & 0 \\
-\delta \epsilon_{w} & 0 & 1+\epsilon_{w} \\
\epsilon_{R} & 0 & 1+k
\end{array}\right] .
\end{aligned}
$$


Matrix $\mathbf{P}_{m}$ can be inverted analytically:

$$
\mathbf{P}_{m}^{-1}=\left[\begin{array}{ccc}
0 & -1 & 1 \\
1 & 1 & -1 \\
0 & 0 & 1
\end{array}\right]
$$

The system (29) can then be rewritten as follows:

$$
\partial_{\hat{t}} \mathbf{X}=\mathbf{N}_{m} \cdot \mathbf{X}
$$

with

$$
\mathbf{N}_{m}=\mathbf{P}_{m}^{-1} \cdot \mathbf{M}_{m}=-\left[\begin{array}{ccc}
\delta \epsilon_{w}+\epsilon_{R} & 0 & k-\epsilon_{w} \\
-\epsilon_{R} & 1+\epsilon_{w} & \epsilon_{w}-k \\
\epsilon_{R} & 0 & 1+k
\end{array}\right]
$$

The characteristic equation of $\mathbf{N}_{m}$ can be written

$$
\begin{gathered}
-\left(\mathbf{x}+1+\epsilon_{w}\right)\left[\mathbf{x}^{2}+\left(1+k+\delta \epsilon_{w}+\epsilon_{R}\right) \mathbf{x}\right. \\
\left.+(1+k)\left(\delta \epsilon_{w}+\epsilon_{R}\right)+\epsilon_{R}\left(\epsilon_{w}-k\right)\right]=0 .
\end{gathered}
$$

The characteristic time scales $\left(\tau_{1}^{m}, \tau_{2}^{m}, \tau_{3}^{m}\right)$ of the linear adjustment of the MLM are the absolute values of the inverses of the three eigenvalues of $\mathbf{N}_{m}$. They can be expressed as follows:

$$
\begin{aligned}
& \tau_{1}^{m}=\left(1+k-\chi_{m}\right)^{-1}, \\
& \tau_{2}^{m}=\left(1+\epsilon_{w}\right)^{-1}, \quad \text { and } \\
& \tau_{3}^{m}=\left(\delta \epsilon_{w}+\epsilon_{R}+\chi_{m}\right)^{-1},
\end{aligned}
$$

with

$$
\begin{aligned}
\chi_{m}= & \frac{1}{2}\left(1+k-\delta \epsilon_{w}-\epsilon_{R}\right) \\
& \cdot\left\{1-\left[1-\frac{4 \epsilon_{R}\left(\epsilon_{w}-k\right)}{\left(1+k-\delta \epsilon_{w}-\epsilon_{R}\right)^{2}}\right]^{1 / 2}\right\} .
\end{aligned}
$$

The characteristic scale $\tau_{2}^{m}$ corresponds to the adjustment by the combination of the surface fluxes (indicated by the 1 since the surface-flux time scale was used to nondimensionalize the equations) and the subsidence $\epsilon_{w}$, and was identified as the "fast" time scale $\tau_{\text {th }}$ by Schubert et al. (1979b) and Bretherton et al. (2010).

The parameter $\chi_{m}$ is small compared to $1+k$ and so that neglecting $\chi_{m}$ only introduces a small error on $\tau_{1}^{m}$ ( $6 \%$ for the reference equilibrium state with $T_{s}=299 \mathrm{~K}$ ) and a slightly larger error on $\tau_{3}^{m}(15 \%$ for the reference equilibrium state with $\left.T_{s}=299 \mathrm{~K}\right)$. We can write

$$
\tau_{1}^{m} \approx(1+k)^{-1}
$$

$$
\begin{aligned}
\tau_{2}^{m} & =\left(1+\epsilon_{w}\right)^{-1}, \quad \text { and } \\
\tau_{3}^{m} & \sim\left(\delta \epsilon_{w}+\epsilon_{R}\right)^{-1},
\end{aligned}
$$

which shows that $\tau_{1}^{m}$ is the time scale of adjustment of the buoyancy through turbulent fluxes at the surface (indicated by the 1) and at cloud base (indicated by $k$ ), and that $\tau_{3}^{m}$ results mostly from the combination of radiation $\epsilon_{R}$ and divergence at the top of the layer $\delta \epsilon_{w}$. While $\tau_{1}^{m}$ is strongly dependent on the closure, $\tau_{3}^{m}$ 's scaling is not; it is similar to the "slow" time scale $\tau_{i}$ in Schubert et al. (1979b) and Bretherton et al. (2010), but it combines the damping effect of radiation to that of the divergence.

For a thin, 500-m-deep boundary layer under a strong inversion, $\tau_{1}^{m}, \tau_{2}^{m}$, and $\tau_{3}^{m}$ correspond to dimensional time scales of 9, 9, and about $50 \mathrm{~h}$. The MLM therefore provides a scale separation between the thermodynamic time scales and the dynamic (or mass) time scale, as pointed out by Schubert et al. (1979b) and Bretherton et al. (2010), and which can alternatively be thought of as the fast processes of turbulence and mean advection of the anomaly and the slow processes associated with radiation and anomalous advection. For a shallowconvective CuBL such as simulated by the XLM with $T_{s}=299 \mathrm{~K}, \tau_{1}^{m}, \tau_{2}^{m}$, and $\tau_{3}^{m}$ correspond to dimensional time scales of 19,16 , and $37 \mathrm{~h}$, so the MLM does not capture the scale separation between the two thermodynamic time scales as clearly as the LES. In the next section, we investigate how the introduction of a vertical structure in the CuBL and the use of the buoyancy closure at cloud base rather than at the inversion change these adjustment time scales in the XLM and explain the early time evolution of the thermodynamical variables.

\section{b. Approximations to the XLM adjustment time scales}

In the linear system of equations [Eq. (23)], the coefficient in the first column of $\mathbf{M}$, which are associated to the perturbations in $\hat{h}$, are small $[o(1)$ while the other coefficients are $O(1)]$. For the reference equilibrium state at $T_{s}=299 \mathrm{~K}, \delta \gamma \epsilon_{w} \approx 0.10$, and $\delta \gamma \epsilon_{w}-(1-\gamma) \epsilon_{R} \approx$ 0.06 . At first order, these coefficients can be neglected, and the matrices $\mathbf{M}$ and $\mathbf{N}$ can be approximated by the following matrices $\mathbf{M}^{o}$ and $\mathbf{N}^{o}$ :

$$
\mathbf{M}^{o}=-\left[\begin{array}{ccc}
0 & 1+\gamma \epsilon_{w} & 0 \\
0 & 0 & 1+\gamma \epsilon_{w} \\
0 & -Q \epsilon_{R} & \epsilon_{b}
\end{array}\right] \text { and }
$$




$$
\mathbf{N}^{o}=-\frac{1}{\tilde{\eta} \nu}\left[\begin{array}{ccc}
0 & \tilde{\eta} \alpha Q\left(1+\gamma \epsilon_{w}\right)-\psi Q \epsilon_{R} & -\tilde{\eta}(1-\alpha Q)\left(1+\gamma \epsilon_{w}\right)+\psi \epsilon_{b} \\
0 & \tilde{\eta} \lambda\left(1+\gamma \epsilon_{w}\right)+\mu Q \epsilon_{R} & \tilde{\eta}(1-\alpha)\left(1+\gamma \epsilon_{w}\right)-\mu \epsilon_{b} \\
0 & -\nu Q \epsilon_{R} & \nu \epsilon_{b}
\end{array}\right],
$$

where $\epsilon_{b}=1+k+\Theta \epsilon_{R}$ is the characteristic response rate of the subcloud-layer buoyancy to a buoyancy perturbation, including the response of the turbulent fluxes at the surface (1) and at cloud base $(k)$, and the radiative contribution associated to the change in $\operatorname{LCL}\left(\Theta \epsilon_{R}\right)$.

The characteristic equation of $\mathbf{N}^{o}$ can be written

$$
\begin{gathered}
-\mathbf{x}\left\{\tilde{\eta} \nu \mathbf{x}^{2}+\left[\nu \epsilon_{b}+\mu Q \epsilon_{R}+\tilde{\eta} \lambda\left(1+\gamma \epsilon_{w}\right)\right] \mathbf{x}\right. \\
\left.+\left(1+\gamma \epsilon_{w}\right)\left[\lambda \epsilon_{b}+(1-\alpha) Q \epsilon_{R}\right]\right\}=0,
\end{gathered}
$$

where $\mathbf{x}_{3}=0$ is an eigenvalue of the matrix that corresponds to an infinite adjustment time scale $\tau_{3}^{o}$, and the two other eigenvalues $\mathbf{x}_{1}$ and $\mathbf{x}_{2}$ are solutions of the quadratic equation in parentheses. Here $\tau_{1}^{o}=\mathbf{x}_{1}^{-1}$ and $\tau_{2}^{o}=\mathbf{x}_{2}^{-1}$ are first-order approximations of the adjustment time scales $\tau_{1}$ and $\tau_{2}$ :

$$
\begin{aligned}
\tau_{1}^{o} & =\frac{\tilde{\eta} \nu}{\nu \epsilon_{b}+\mu Q \epsilon_{R}-\chi} \quad \text { and } \\
\tau_{2}^{o} & =\frac{\tilde{\eta} \nu}{\tilde{\eta} \lambda\left(1+\gamma \epsilon_{w}\right)+\chi},
\end{aligned}
$$

with

$$
\begin{aligned}
\chi= & \frac{1}{2}\left[\nu \epsilon_{b}+\mu Q \epsilon_{R}-\tilde{\eta} \lambda\left(1+\gamma \epsilon_{w}\right)\right] \\
& \cdot\left(1-\left\{1-\frac{4 \tilde{\eta}[(1-\alpha) \nu-\lambda \mu]\left(1+\gamma \epsilon_{w}\right) Q \epsilon_{R}}{\left[\nu \epsilon_{b}+\mu Q \epsilon_{R}-\tilde{\eta} \lambda\left(1+\gamma \epsilon_{w}\right)\right]^{2}}\right\}^{1 / 2}\right) .
\end{aligned}
$$

The first-order time scales $\tau_{1}^{o}$ and $\tau_{2}^{o}$ are shown in Fig. 5 (open circles): they match the exact time scales $\tau_{1}$ and $\tau_{2}$ very well. The parameter $\chi$ is small compared to $\nu \epsilon_{b}+$ $\mu Q \epsilon_{R}$ so that neglecting $\chi$ only introduces a small error on $\tau_{1}^{o}\left(6 \%\right.$ for the reference equilibrium state with $T_{s}=$ $299 \mathrm{~K}$ ). The error is more significant for $\tau_{2}^{o}$ (27\% for the reference equilibrium state with $T_{s}=299 \mathrm{~K}$ ). Figure 5 also shows the approximations of $\tau_{1}^{o}$ and $\tau_{2}^{o}$ computed with $\chi$ set to zero (dotted lines). This approximation is fair for $\tau_{1}$, and although it is not as good an approximation for $\tau_{2}$ as for $\tau_{1}$, it scales fairly well with $\tau_{2}$. Replacing $\epsilon_{b}$ by its expression, we can write the following approximations for $\tau_{1}$ and $\tau_{2}$ :

$$
\begin{aligned}
& \tau_{1} \approx \tilde{\eta}\left[1+k+\left(\Theta+\frac{\mu}{\nu} Q\right) \epsilon_{R}\right]^{-1} \text { and } \\
& \tau_{2} \sim \frac{\nu}{\lambda}\left(1+\gamma \epsilon_{w}\right)^{-1} .
\end{aligned}
$$

Equation (47) shows that the fastest adjustment time scale $\tau_{1}$ results from the buoyancy adjustment of the subcloud layer [and scales with its depth $\tilde{\eta}$ similarly to the surface-flux time scale proposed by Betts (1993)] through turbulent fluxes $(1+k)$ and a radiative contribution associated to the perturbation of the subcloudlayer depth $\left[(\Theta+\mu / \nu Q) \epsilon_{R}\right]$. Equation (48) shows that the second-fastest adjustment time scale $\tau_{2}$ is essentially controlled by the combined damping effect of the surface fluxes (1) and vertical advection of the anomalies $\left(\gamma \epsilon_{w}\right)$ on the whole $\mathrm{CuBL}$, with a multiplicative factor $\nu / \lambda$ resulting from the mixing-line hypothesis: $-\lambda$ is the rate of increase of the CuBL heat content with $h$, and $\tilde{\eta} \nu$ is the determinant of matrix $\mathbf{P}$.

Compared to the scalings of the MLM characteristic time scales $\tau_{1}^{m}$ and $\tau_{2}^{m}$ [see Eqs. (39) and (40)], the XLM $\tau_{1}$ is shorter because the closure is applied to the top of the subcloud layer (thus the factor $\tilde{\eta}$ ) that follows the LCL (thus the terms in $Q$ and $\Theta$ ), and both effects reduce the time scale $\tau_{1}$ compared to $\tau_{1}^{m}$. The time scale $\tau_{2}$ is lengthened because of the introduction of a vertical structure in the $\mathrm{CuBL}$ that appears through the ratio $\nu / \lambda>1$, which results from the redistribution of heat and humidity imposed by the mixing-line constraints on their profiles, and through the coefficient $\gamma<1$, which reduces the effect of the vertical advection. As a result, the XLM provides an additional scale separation compared to the well-mixed case between the buoyancy adjustment of the subcloud layer and the thermodynamic adjustment of the whole CuBL. The residual adjustment of the CuBL depth is slower than in the well-mixed case so that its time scale is still separated from the thermodynamic adjustment time scales.

\section{Summary and conclusions}

The adjustment of the trade wind boundary layer to an abrupt SST increase as represented by large-eddy simulation involves three characteristic scales: one for the adjustment of the near-surface (virtual potential) 
temperature associated with a decrease in near-surface humidity, one for the adjustment of the near-surface humidity, and one for the adjustment of the CuBL depth.

Bellon and Stevens (2012) showed that the XLM bulk model reproduces the sensitivity of the equilibrium CuBL to SST, as represented by LES. The present work also shows that the XLM captures some of the fundamental processes of the transient behavior of the LES in response to SST change, and in particular the characteristic time scales and the nonmonotonic evolution of the near-surface humidity. Compared to the MLM (and apparently other mixed-layer models used in previous studies), the XLM provides an additional scale separation between the fast buoyancy-driven adjustment of the subcloud temperature and the slower adjustment of the humidity and residual temperature perturbation, and it conserves the scale separation between the adjustment of thermodynamical variables and that of the CuBL depth.

In the fastest linear mode of the XLM, the perturbations of subcloud humidity and subcloud temperature have opposite signs. This explains why the humidity initially decreases in the nonlinear simulations, which is physically counterintuitive. In this mode, all the processes interact constructively to adjust one of the smallest reservoir in the system (i.e., the subcloud reservoir of buoyancy). This type of behavior has been documented in simple climate models (e.g., Geoffroy et al. 2013), in which the first reservoir to be adjusted is the one with the smallest heat capacity, to the expense of the adjustment of other reservoirs. In the case of the XLM (and presumably the LES), the perturbation of the size of the reservoir associated with the fastest mode is also negative, accelerating the adjustment.

Although the present results do not incorporate the effects that may arise by allowing radiation to act interactively, or precipitation to develop, they do shed some light on what can be expected from much less idealized simulations such as Lagrangian experiments with a progressive warming of the SST. They suggest that the fastest mode of adjustment effectively buffers changes in sea surface temperatures but accentuates changes in surface saturation humidity through the rapid transport of warmer, drier air into the subcloud layer in response to increases in sea surface temperatures.

Acknowledgments. The authors thank A. K. Betts, P. N. Blossey, and a third anonymous reviewer for their useful comments. Figure 3 was suggested by P. N. Blossey. Thanks are due to C. Jones for his careful reading and corrections. The authors are also grateful to the Centre National de la Recherche Scientifique and the Max Planck Society for the Advancement of Science for supporting their research.

\section{REFERENCES}

Albrecht, B. A., C. S. Bretherton, D. Johnson, W. H. Schubert, and A. S. Frisch, 1995: The Atlantic Stratocumulus Transition Experiment-ASTEX. Bull. Amer. Meteor. Soc., 76, 889-904.

Bellon, G., and B. Stevens, 2012: Using the sensitivity of large-eddy simulations to evaluate atmospheric boundary layer models. J. Atmos. Sci., 69, 1582-1601.

Betts, A. K., 1993: Mixing line parameterizations for cloudy boundary layers. Proc. Workshop on the Parameterization of Cloud Topped Boundary Layers, Reading, United Kingdom, ECMWF/GCSS, 239-257.

—, and W. Ridgway, 1988: Coupling of the radiative, convective, and surface fluxes over the equatorial Pacific. J. Atmos. Sci., 45, 522-546.

Bretherton, C. S., and S. Park, 2008: A new bulk shallow-cumulus model and implications for penetrative entrainment feedback on updraft buoyancy. J. Atmos. Sci., 65, 2174-2193.

, S. K. Krueger, M. C. Wyant, P. Bechtold, E. V. Meijgaard, B. Stevens, and J. Teixeira, 1999: A GCSS boundary-layer cloud model intercomparison study of the first ASTEX Lagrangian experiment. Bound.-Layer Meteor., 93, 341-380.

— - J. Uchida, and P. N. Blossey, 2010: Slow manifolds and multiple equilibria in stratocumulus-capped boundary layers. J. Adv. Model. Earth Syst., 2 (14), 20 pp. [Available online at http://james.agu.org/index.php/JAMES/article/viewArticle/ v2n14.]

Geoffroy, O., D. Saint-Martin, D. J. L. Olivié, A. Voldoire, G. Bellon, and S. Tytéca, 2013: Transient climate response in a two-layer energy-balance model. Part I: Analytical solution and parameter calibration using CMIP5 AOGCM experiments. J. Climate, 26, 1841-1857.

Lilly, D. K., 1968: Models of cloud-topped mixed layers under a strong inversion. Quart. J. Roy. Meteor. Soc., 94, 292-309.

Nuijens, L., and B. Stevens, 2012: The influence of wind speed on shallow marine cumulus convection. J. Atmos. Sci., 69, 168-184.

Sandu, I., and B. Stevens, 2011: On the factors modulating the stratocumulus to cumulus transitions. J. Atmos. Sci., 68, 18651881.

Savic-Jovcic, V., and B. Stevens, 2008: The structure and mesoscale organization of precipitating stratocumulus. J. Atmos. Sci., 65 , $1587-1605$

Schubert, W. H., J. S. Wakefield, E. J. Steiner, and S. K. Cox, 1979a: Marine stratocumulus convection. Part I: Governing equations and horizontally homogeneous solutions. J. Atmos. Sci., 36, 1286-1307.

,,$---1979 \mathrm{~b}$ : Marine stratocumulus convection. Part II: Horizontally inhomogeneous solutions. J. Atmos. Sci., 36, 1308-1324.

Stevens, B., and Coauthors, 2005: Evaluation of large-eddy simulations via observations of nocturnal marine stratocumulus. Mon. Wea. Rev., 133, 1443-1462.

Tompkins, A. M., and G. C. Craig, 1998: Time-scales of adjustment to radiative-convective equilibrium in the tropical atmosphere. Quart. J. Roy. Meteor. Soc., 124, 2693-2713. 
Copyright of Journal of the Atmospheric Sciences is the property of American Meteorological Society and its content may not be copied or emailed to multiple sites or posted to a listserv without the copyright holder's express written permission. However, users may print, download, or email articles for individual use. 